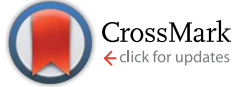

Cite this: RSC Adv., 2016, 6, 111060

Received 22nd September 2016 Accepted 13th November 2016

DOI: $10.1039 / c 6 r a 23620 k$

www.rsc.org/advances

\section{Dual encapsulation of hydrophobic and hydrophilic drugs in PLGA nanoparticles by a single-step method: drug delivery and cytotoxicity assays $\uparrow$}

Laura Español, ${ }^{\text {aa }}$ Ane Larrea, ${ }^{\text {b }}$ Vanesa Andreu, ${ }^{\text {b }}$ Gracia Mendoza, ${ }^{\text {b }}$ Manuel Arruebo, ${ }^{\text {bc }}$ Victor Sebastian, *bc María S. Aurora-Prado, ${ }^{a}$ Erika R. M. Kedor-Hackmann, ${ }^{a}$

Maria Ines R. M. Santoro ${ }^{a}$ and Jesus Santamariabc

Dual drug encapsulation in biodegradable nanoparticles is always challenging and often requires strenuous optimization of the synthesis-encapsulation processes. This becomes even more difficult when the simultaneous encapsulation of molecules of different polarity is sought. Here we present a modified emulsification-evaporation process to produce polymeric nanoparticles (NPs) made of the biocompatible and biodegradable polymer poly(lactic-co-glycolic acid) (PLGA) and co-encapsulating simultaneously two different drugs, the hydrophobic dexamethasone (DX) and the hydrophilic diclofenac sodium (DS). Three independent processing parameters were systematically modified to promote the incorporation of the different-polarity drugs into PLGA and to control the particle size under $150 \mathrm{~nm}$. The careful selection of the appropriate solvents (ethyl acetate and methanol) was a key requirement for the successful encapsulation of DX and DS. DS and DX release kinetics as well as cytotoxicity assays underlined the therapeutic potential of the dual encapsulation strategy.

\section{Introduction}

Nanomedicine is the branch of nanotechnology that pursues the development of new medical tools to provide significant advances in the diagnosis and treatment of many diseases. ${ }^{1}$ In that sense, nanoparticle-based drug delivery systems show many advantages, including improving the solubility of poorly water-soluble drugs, modifying drug pharmacokinetics, increasing the drug half-life (by reducing recognition and immunogenicity), targeting drugs to a specific diseased site (therefore reducing side effects), improving bioavailability and retention times, and reducing the drug metabolism. ${ }^{2,3}$

In recent years, a variety of scenarios requiring combination drug therapy has stimulated research to develop nanoparticulated vehicles that can deliver more than one drug in a controlled manner. These nanosystems promote an effective treatment with the advantage of a drug synergistic effect

${ }^{a}$ Faculty of Pharmaceutical Sciences, University of Sao Paulo, 05508-000 Sao Paulo, Brazil

${ }^{b}$ Department of Chemical Engineering, Aragon Institute of Nanoscience (INA), University of Zaragoza, Campus Río Ebro-Edificio I+D, C/Poeta Mariano Esquillor S/ N, 50018 Zaragoza, Spain. Tel: +34 876555441. E-mail: victorse@unizar.es; jesus. santamaria@unizar.es

${ }^{c}$ CIBER de Bioingeniería, Biomateriales y Nanomedicina (CIBER-BBN), C/Monforte de Lemos 3-5, Pabellón 11, 28029 Madrid, Spain

$\dagger$ Electronic supplementary information (ESI) available: Validation of HPLC and MEKC methods, supporting information of the $2^{3}$ factorial design model, SEM images and statistics data. See DOI: $10.1039 / \mathrm{c} 6 \mathrm{ra} 23620 \mathrm{k}$ through controlled combinatorial drug delivery. ${ }^{4}$ In spite of this, a myriad of studies investigate single-drug incorporation into carrier nanoparticles, while nanoparticles loaded with multiple drugs are reported scarcely. ${ }^{4,5}$ One of the main hurdles in combination drug therapy is the difficulty in coencapsulating hydrophobic and hydrophilic drugs in the same carrier, due to the solubility problems inherent to the simultaneous encapsulation of drugs with different polarities. In this regard, Zhang et al. ${ }^{6}$ co-encapsulated chemotherapeutics with distinct water solubility properties, docetaxel as a model of hydrophobic drug and doxorubicin hydrochloride (DOX) as a model of hydrophilic drug. They used the biocompatible and biodegradable PLGA- $b$-PEG copolymer for the encapsulation using the nanoprecipitation method, and showed that both drugs could be released from the conjugates over time. In another study, Song et al. ${ }^{5}$ studied the co-encapsulation of two drugs with different polarities: vincristine sulfate (VCR) and quercetin (QC), hydrophilic and hydrophobic molecules, respectively. The $\mathrm{O} / \mathrm{W}$ emulsion solvent-evaporation method with a mixture of acetone and dichloromethane $(1: 2)$ was used to promote drug loading. However, in this case, very low drug loadings were reported: $0.0037 \pm 0.0001 \%$ for VCR and $1.36 \pm$ $0.12 \%$ for QC.

In the case of acute or chronic diseases where pain and inflammation are present, two main therapeutic approaches are currently adopted: the use of non-steroidal anti-inflammatory drugs (NSAIDs), which are generally short-acting medical products, and corticosteroids (long-acting formulations). The 
NSAIDs are good candidates for the elaboration of controlled release preparations, particularly through the oral route ${ }^{7}$ and have both anti-inflammatory and analgesic effects due to the inhibition of the pro-inflammatory enzyme cyclooxygenase (COX). However, the chronic use of this group of drugs is associated with various adverse gastrointestinal effects, mainly development of ulceration and/or bleeding in the gastrointestinal tract. ${ }^{8}$ Therefore, any new drug delivery system must satisfy strict controls over the drug release kinetics to avoid overdosing at any stage.

Diclofenac, a NSAID, is usually administered as sodium or potassium salts showing potent anti-inflammatory, analgesic and antipyretic properties. ${ }^{7}$ Diclofenac sodium (DS) is used in a varied number of clinical disorders, including rheumatoid arthritis, osteoarthritis, ankylosing spondylitis, gout, dysmenorrhea, dental pain and headache. ${ }^{9,10}$ Glucocorticoids are the most commonly used anti-inflammatory and immunosuppressive drugs in the treatment of a wide range of rheumatic and other inflammatory diseases. ${ }^{\mathbf{1 1}}$ Dexamethasone (DX) is a glucocorticoid clinically used as an anti-inflammatory and immunosuppressive agent. However, several side effects, such as hypertension, hydroelectrolytic disorders, hyperglycemia, peptic ulcers and glucosuria restrict its use in prolonged therapies. ${ }^{12}$ Both drugs, DX and DS, are used to control inflammation and alleviate pain in patients with osteoarthritis, reducing COX activity by following two different mechanisms. When liposomal formulations for DX and DS (alone or in combination) were investigated, it was clearly demonstrated that their co-administration retained their biological activities and had the most favorable therapeutic response compared to the application of both drugs alone. ${ }^{13}$ However, liposomal formulations still show insufficient drug entrapment and low stability.

A vector capable of simultaneous delivery of DX and DS would present significant therapeutic advantages compared to the effects of identical concentrations of the same drugs administered independently. For instance, the coadministration of low doses of DX and DS on carrageenan strongly reduces both peripheral inflammation and the associated spinal expression of c-Fos, an indicator of nociceptive transmission at the spinal level. ${ }^{\mathbf{1 4 , 1 5}}$ A prospective, randomized, open-label pilot study was carried out to assess the effects of a combination treatment with dexamethasone and potassium diclofenac or acetaminophen in comparison with potassium diclofenac alone in postoperative pain, swelling, and trismus after surgical removal of third molars. ${ }^{16}$ In this study, the concomitant treatment of dexamethasone and potassium diclofenac provided significant pain relief and reduced swelling and postsurgical pain compared to dexamethasone and acetaminophen or monotherapy with diclofenac. The apparent interactions between the mechanisms of action of NSAIDs and steroids suggest that co-therapy may produce beneficial inflammatory and pain relief in the absence of excessive side effects. ${ }^{17}$ This provides a strong motivation for the development of carriers to co-administer appropriate doses of both drugs.

Biodegradable polymers such as poly(D,L-lactic-co-glycolic acid) (PLGA) and poly(D,L-lactic acid) (PLA) have been commonly used as nanoparticulated materials to encapsulate a variety of therapeutic compounds including chemotherapeutic drugs, anti-inflammatories, peptides and proteins. ${ }^{18-20}$ PLGA is a commercially available, biodegradable and biocompatible copolymer approved by the FDA (Food and Drugs Administration, USA) in many medical devices and formulations. Drugs entrapped in PLA- or PLGA-based polymeric devices are released by diffusion through the polymeric matrix, by erosion of the polymer, due to the hydrolysis of its ester bonds, or by the combination of both mechanisms. ${ }^{21,22}$ PLGA biodegradation produces natural metabolites (lactic and glycolic acids), which are eliminated from the body by the Krebs cycle under physiological conditions. ${ }^{23}$

The strategies employed to co-encapsulate two or more drugs into a single carrier include physical loading, chemical conjugation and covalent linkage between the polymer and the therapeutic agents. On the other hand, PLGA nanoparticles (NPs) can be prepared by emulsion solvent evaporation, nanoprecipitation, solvent displacement and salting-out. Considering that physical encapsulation is a drug loading strategy that has been widely used ${ }^{4}$ and taking into account the different polarity of DX and DS, we decided to investigate a single emulsification evaporation method as a promising alternative for the combined encapsulation of drugs in a single delivery vector. The expected advantages of this encapsulation are: (1) localized administration of two drugs with different action mechanisms with potential synergistic therapeutic effect. (2) Reduction of side effects due to the administration of the appropriate dose in a controlled manner. In addition, drug encapsulation in a polymeric nanocarrier protects the drugs from premature degradation, improves the solubility of hydrophobic molecules, lowers the toxicity risks, and enhances drug efficacy, specificity, tolerability and therapeutic index. ${ }^{\mathbf{2 4}}$

In this work, we have optimized a single emulsion encapsulation methodology through a systematic assessment of independent processing parameters with the objective of maximizing the loading of both hydrophobic (DX) and hydrophilic (DS) drugs in the same biodegradable matrix. Cytotoxicity assessment of the produced nanoparticles and drug release studies were also carried out to evaluate the performance of the developed vector.

\section{Materials and methods}

\subsection{Materials}

The polymer poly(D,L-lactic acid/glycolic acid) 50/50 (PLGA, MW: 38-54 kDa) Resomer® RG 504 was purchased from Boehringer Ingelheim (Ingelheim, Germany). Sigma Aldrich provided the HPLC-grade solvents ethyl acetate, methanol and acetonitrile, as well as diclofenac sodium, dexamethasone Pluronic $®$ F68 (average MW $8350 \mathrm{Da})$, sodium tetraborate (99\%), sodium hydroxide (>97\%), sodium dodecyl sulfate (98\%) and the electronic microscopy contrast agent phosphotungstic acid (reagent grade).

\subsection{Preparation and characterization of the formulations}

Experimental design. Minitab® software version 15 was used to adapt the $2^{3}$ factorial design to optimize the loading in the 
DX-DS-loaded PLGA NPs (DX-DS/PLGA-NPs). The independent variables for optimization were selected according to published data as follows: (a) drug: polymer ratio (X1 = PLGA), (b) concentration of Pluronic as surfactant (X2 = PLUR) and (c) sonication time $(\mathrm{X} 3=\mathrm{ST})$. The selected responses were $(1)$ the mean particle size of the DX-DS/PLGA-NPs (Y1), (2) the zeta potential of the DX-DS/PLGA-NPs (Y2), (3) the encapsulation efficiency (Y3). Each independent variable was given a high and low level value (Table 1$)$.

Preparation of DX-DS/PLGA nanoparticles. The DX/DS loaded nanoparticles were prepared using the single emulsification/evaporation method $^{25}$ with slight modifications. Briefly, 50-100 mg of PLGA Resomer 504 and 150$250 \mathrm{mg}$ of surfactant Pluronic F-68 were dissolved in $4 \mathrm{~mL}$ of ethyl acetate (EA) and both drugs (diclofenac sodium and dexamethasone) were dissolved in $1 \mathrm{~mL}$ of a solution consisting of ethyl acetate : methanol (ratio $9: 1 \mathrm{v} / \mathrm{v}$ ). Then, this organic phase was emulsified with the aqueous phase $10 \mathrm{~mL}$ of Milli-Q water $(\mathrm{pH}=3.8$ adjusted with $0.1 \mathrm{M}$ hydrochloric acid) by sonication in an ice bath for 25-45 seconds using an ultrasonic probe of $12 \mathrm{~mm}$ diameter and setting the sonifier at a $40 \%$ of amplitude (Digital Sonifier 450, Branson, USA). The resulting o/w-emulsion was maintained under constant stirring for 6 hours to allow the total evaporation of the organic solvent and simultaneously induce the precipitation of the PLGA NPs. Drug loaded NPs were then centrifuged (Spectrafuge 24D, Labnet) at $3000 \mathrm{~g}$ for $15 \mathrm{~min}$, the supernatant was discarded and the pellet was resuspended in a solution of the cryoprotector mannitol $5 \mathrm{wt} \%$. The washed NPs were finally frozen in liquid nitrogen and lyophilized at $-40{ }^{\circ} \mathrm{C}$ and $0.05 \mathrm{mbar}$ for $24 \mathrm{~h}$ (Freezone 4.5 model 77510, Labconco).

\subsection{Physicochemical characterization of DX-DS/PLGA nanoparticles formulations}

Dynamic light scattering (DLS). The hydrodynamic diameter of the resulting nanoparticles was determined by dynamic light scattering measurements. Nanoparticles were suspended in 2 $\mathrm{mL}$ of distilled water and sonicated before measurements. Nanoparticle size distribution was determined using a Brookhaven 90 Plus (Holtsville, NY) equipment. Each sample was measured three times.

Zeta potential measurements. The zeta potential of the drug loaded nanoparticles, directly related to the surface charge density, was measured by using a Brookhaven 90 Plus equipment. Zeta potential values were determined using $100 \mu \mathrm{L}$ of the nanoparticle suspension and adding $1.5 \mathrm{~mL}$ of $1 \mathrm{mM} \mathrm{KCl}$ at $\mathrm{pH}$ 7.4. Three repeated measurements of the $Z$ potential were performed for each individual experiment.

Morphological studies. The morphological characterization of the resulting PLGA nanoparticles was performed at the LMAINA-UNIZAR facilities by Scanning Electron Microscopy in a SEM (Inspect ${ }^{\mathrm{TM}}$ F50 Scanning Electron Microscope, Holland, FEI, accelerating voltage: $200 \mathrm{~V}$ to $30 \mathrm{kV}$, equipped with a Schottky Field Emission source) and a Transmission Electron Microscope TEM (TECNAI FEI T20) operating at an acceleration voltage $200 \mathrm{kV}$. The nanoparticle samples were stained with $7 \%$ $(\mathrm{w} / \mathrm{v})$ phosphotungstic acid as a negative contrast to facilitate observation. The samples prepared for SEM characterization were deposited on a silicon wafer and coated with platinum to minimize surface charge, prior examination. The TEM samples were placed on a carbon-coated grid and dried at room temperature for observation.

X-ray diffractometry (XRD). X-ray diffraction studies were realized in a X-ray diffractometer (RIGAKU, D/max 2500) equipped with a rotating anode. The diffractometer was used at $40 \mathrm{kV}$ and $80 \mathrm{~mA}$ with a $\mathrm{Cu}$ anode and a graphite monochromator to select the $\mathrm{CuK} \alpha$ radiation. Measurement conditions were $2 \theta 5^{\circ}$ to $60^{\circ}$, step $=0.03^{\circ}, t=1 \mathrm{~s}$ per step.

Fourier-transform infrared spectroscopy (FT-IR). The potential chemical interactions between the encapsulated drugs and the host PLGA copolymer, were studied by Fourier Transformed Infra-Red Spectroscopy (FTIR). The infrared (IR) spectra of free powdered drugs (dexamethasone and diclofenac sodium), a physical mixture of the drugs and the polymer and drug-loaded nanoparticles were measured on a VERTEX 70 Series FT-IR Spectrometer (Bruker Biosciences, Spain).

Drug loading and entrapment efficiency. To measure the encapsulation efficiency (EE), lyophilized drug-loaded nanoparticles were weighed and dissolved in water: acetonitrile $(6: 5, \mathrm{v} / \mathrm{v})$ and, then, extracted with methanol. After centrifugation at $5000 \mathrm{~g}$ for $10 \mathrm{~min}$, the supernatant was filtered through a $0.20 \mu \mathrm{m}$ Teflon filter and the DX and DS content was determined by high performance liquid chromatography (HPLC) and micellar electrokinetic chromatography (MEKC), a mode of capillary electrophoresis (CE) technique.

The drug encapsulation is expressed both as drug loading (DL) and entrapment efficiency (EE), represented by eqn (1) and (2), respectively.

$$
\mathrm{DL}(\%)=\frac{\text { mass of drug in NPs }}{\text { total NPs mass }} \times 100
$$

Table 1 Independent and dependent variables used for the $2^{3}$ factorial design

\begin{tabular}{lllll}
\hline & & Level & & \\
\cline { 3 - 4 } & Independent variables & Low & High & Dependent variables \\
\hline X1 & Drug : polymer ratio (\% PLGA w/w) & $1: 5(1 \%) 50 \mathrm{mg} \mathrm{PLGA}$ & $1: 10(2 \%)$ 100 mg PLGA & Particle size \\
$\mathrm{X} 2$ & Pluronic concentration (\% w/w) & 3 & 5 & Zeta potential \\
$\mathrm{X} 3$ & Sonication time (s) & 25 & 45 & Encapsulation efficiency
\end{tabular}




$$
\mathrm{EE}(\%)=\frac{\text { mass of drug in NPs }}{\text { total mass of drug }} \times 100
$$

High performance liquid chromatography methodology (HPLC). The analyses were made in an HPLC-Waters Instrument 2690 (Alliance, USA) at $260 \mathrm{~nm}$. A Phenomenex ${ }^{\circledR}$ Kinetex C18 $75 \times 4 \mathrm{~mm}, 2.6 \mu \mathrm{m}$ column was used. The solvent system consisted of acetonitrile: water (pH 3) 50 : 50, v/v. An injection volume of $20 \mu \mathrm{L}$ and a flow rate of $0.4 \mathrm{~mL} \mathrm{~min}^{-1}$ at $40{ }^{\circ} \mathrm{C}$ were used. The DX and DS were detected at a retention time of $1.9 \mathrm{~min}$ and $5.1 \mathrm{~min}$, respectively (Fig. S1†). The HPLC method was previously validated (Fig. S1 $\dagger$ ).

Micellar electrokinetic chromatography methodology (MECK). MEKC studies were made on a Beckman PACE/MDQ equipment (Beckman Instruments, CA, USA) equipped with an on-column diode-array detection (DAD) system. Software for data acquisition and treatment (32 Karat TM software) was used. An uncoated fused-silica capillary column (Polymicro Technologies, Phoenix, AZ, USA) was used with $50 \mu \mathrm{m}$ ID $\times$ $32 \mathrm{~cm}$ total length $(20 \mathrm{~cm}$ to detector). The capillaries were thermostatted at $25{ }^{\circ} \mathrm{C}$ by cooling liquid circulation. Samples were introduced via hydrodynamic injection by applying $0.5 \mathrm{psi}$ for $3 \mathrm{~s}$. The running voltage was $+28 \mathrm{kV}$ and the detection wavelength was set at $240 \mathrm{~nm}$. The electrolyte was constituted of $10 \%(\mathrm{v} / \mathrm{v})$ methanol, $40 \mathrm{mM}$ sodium dodecyl sulfate and $20 \mathrm{mM}$ sodium tetraborate buffer, at a $\mathrm{pH}$ of 9.2 was adjusted with $0.1 \mathrm{M}$ sodium hydroxide. The electrolyte buffer was degassed in an ultrasonic bath and filtered through a $0.22 \mu \mathrm{m}$ membrane filter (Millipore, São Paulo, Brazil) before use. The CE technique was developed and validated for the quantitative determination of diclofenac sodium and dexamethasone (Fig. $\mathrm{S} 2 \dagger$ ).

Drug release. The release studies were carried out using a dialysis-based procedure. The DX-DS/PLGA nanoparticles in suspension $\left(500 \mu \mathrm{L}\right.$ with $\left.0.22 \mathrm{~g} \mathrm{~mL}^{-1}\right)$ were injected into a Slide-ALyzer ${ }^{\circledR}$ dialysis cassette (10k molecular weight). These cassettes were immersed in $40 \mathrm{~mL}$ of PBS in an incubator at $37^{\circ} \mathrm{C}$ under stirring at $300 \mathrm{rpm}$. Samples $(40 \mathrm{~mL})$ of the buffer were collected two hours later, daily for one week, and each time replaced with an equivalent volume of fresh medium. The drug concentration was measured using HPLC as previously described.

\subsection{Biocompatibility studies}

Cell culture and treatments. Human dermal fibroblasts (Lonza, Belgium) were cultured in DMEM high glucose (DMEM w/stable Glutamine, BioWest, France), supplemented with $10 \%$ $\mathrm{v} / \mathrm{v}$ fetal bovine serum (Gibco, UK), antibiotics (100 U penicillin and $100 \mu \mathrm{g} \mathrm{mL}{ }^{-1}$ streptomycin; Lonza, Belgium) and amphotericin $\mathrm{B}\left(1.5 \mu \mathrm{g} \mathrm{mL}^{-1}\right.$; Lonza, Belgium) and maintained in humidified atmosphere at $37{ }^{\circ} \mathrm{C}$ and $5 \% \mathrm{CO}_{2}$.

Three different experimental groups were assessed in biocompatibility assays: (a) free DX and DS, (b) DX-DS/NPs and (c) empty NPs. Prior to the addition to the cells, NPs were sterilized in an ethanol atmosphere and redispersed in sterile PBS. Then, NPs and drugs solutions were added to the fibroblasts (0.01-1 $\mathrm{mg} \mathrm{mL}^{-1}$ ) and incubated for $24 \mathrm{~h}$ at $37{ }^{\circ} \mathrm{C}$ and $5 \% \mathrm{CO}_{2}$.
Cell viability assay. Cytotoxicity of drugs and NPs was evaluated through the Alamar Blue ${ }^{\mathrm{TM}}$ assay (Invitrogen, US) in which non-fluorescent resazurin is reduced into the fluorescent resorufin by metabolically active fibroblasts. Cells were seeded at a density of 6000 cells per well in a 96-well plate, treated with NPs or drugs as described above and the reagent $(10 \%)$ added following incubation for $4 \mathrm{~h}\left(37^{\circ} \mathrm{C}, 5 \% \mathrm{CO}_{2}\right)$. The fluorescence $(535 / 590 \mathrm{~nm}$ ex/em) was assessed in a multimode Synergy HT Microplate Reader (Biotek, US). Values obtained in the absence of drugs or NPs were considered as $100 \%$ of viability. The percentages given are the average of five measurements.

Cell apoptosis analysis by flow cytometry. The evaluation of the effect of NPs or drugs treatment on cell membrane for $24 \mathrm{~h}$ at a subcytotoxic concentration was performed by the analysis of apoptosis by flow cytometry. After treatment, fibroblasts were collected in PBS and a double staining with annexin V-FITC and propidium iodide was performed. This staining consists of an initial treatment of fibroblasts suspensions with annexin VFITC, followed by PBS washing and the addition of a solution containing annexin V binding buffer, annexin V-FITC and propidium iodide. Annexin $\mathrm{V}$ binding buffer was again added after incubation for 15 minutes and samples were analyzed in a FACSARIA BD equipment and FACSDIVA BD software (Cell Separation and Cytometry Unit, CIBA, IIS Aragon, Spain). As background samples, non-treated fibroblasts were used to determine the basal level of apoptosis and necrosis.

Study of cell cycle distribution by flow cytometry. The distribution of cell cycle and the effects of NPs or drugs on fibroblasts nuclei were evaluated by flow cytometry. Fibroblasts were treated as previously described at a subcytotoxic concentration of NPs or drugs. After $24 \mathrm{~h}$, cells were collected in PBS and fixed in $70 \%$ ice-cold ethanol to be further incubated at $4{ }^{\circ} \mathrm{C}$ for $24 \mathrm{~h}$. Then, DNA was stained with propidium iodide and RNase A for 30 minutes and cell cycle phases were analyzed in a FACSArray BD equipment and the MODIFIT 3.0 Verity software. Untreated samples were also analyzed to study the basal distribution of cell cycle phases.

Statistical analysis and optimization procedure. The experimental measurements input parameters were analyzed by using the Minitab® 15 software. The ANOVA analysis for the dependent variables of each effect where the statistical significance was tested was analyzed by comparing the mean square against an estimate of the experimental error (Table S1 $\dagger$ ). The statistical analysis provided the relationship between the dependent variables and independent variables in form of the polynomial equations showed in Table $\mathrm{S} 2 . \dagger$ The criteria for optimizing the DX-DS/PLGA NPs formulation were: reduced particle size, high zeta potential and high DL\% and EE\% (Table S2 $\dagger$ ). Fig. S3† depicts the Pareto chart showing the effects of PLGA concentration (X1), Pluronic concentration (X2) and time of sonication (X3) on particle size, zeta potential, EE\% DX and EE\% DS. All data were expressed as mean $\pm \mathrm{SE}$ (standard error of the mean). Student's test was performed on the data to assess the statistical significance of the differences in particle size and entrapment efficiency percentages between the different nanoparticle 
formulations while ANOVA test was developed to study the significant differences in the cell viability assay (StataSE 12 statistical software). Differences were considered to be statistically significant at a level of $p \leq 0.05$.

\section{Results and discussion}

\subsection{Effect of preparation variables on dexamethasone- diclofenac sodium loaded PLGA nanoparticles formulation}

The O/W single-emulsion solvent-evaporation method was successfully used for preparing a wide variety of DX-DS/PLGA-NP formulations. We used a modification of the standard single emulsion methodology consisting in the use of two miscible solvents with different polarity (ethyl acetate and methanol) to promote the co-encapsulation of two drugs having different polarities. As explained above, the effect of three synthesis variables on the mean diameter, surface charge and EE of DX and DS of the resulting nanoparticles was investigated. A set of eight different formulations was prepared and characterized according to the $2^{3}$ factorial design. This factorial design with the different formulations is given in Table 2 . The effects of drug/polymer ratio, surfactant concentration and time of sonication were investigated using the aforementioned full factorial design (Fig. S5-S7†). The PLGA content was found to have the strongest influence on those variables followed by the time of sonication (Table S3, Fig. S3 and S4 $\dagger$ ).

Surfactant concentration and sonication time. Regarding the sonication time, it is obvious from the data given in Table 2 that increasing the sonication time resulted in a smaller mean NPs sizes. This phenomenon was previously reported and can be rationalized as the increase in the sonication time increases the number of collapsing bubbles during the emulsification and hence the shear rate required for achieving a high dispersion. ${ }^{26}$ Although it is known that prolonged sonication may produce large nanoparticles due to a de-emulsification process, ${ }^{26}$ this was not observed in this study. On the other hand, the time of emulsification does not have a significant effect in the drug entrapment efficiency. Our statistical analysis also indicated the lack of effect of surfactant concentration did not play any role on the variables studied. It is well-known that at high surfactant concentration, the solvent/water interfacial tension is reduced and the emulsion size decreases. However, in this study no significant differences were obtained, and we can conclude that, within the range of conditions used, surfactant concentration has a weaker influence compared to the one exerted by the other variables studied.

PLGA concentration in the organic phase. The morphological characterization of PLGA nanoparticles was carried out by DLS and SEM. The scanning electron microscopy images of the drug-loaded nanoparticles revealed their regular spherical shape (Fig. S8†). Generally, their surface morphology was smooth without any noticeable pinholes or defects. The size distribution of all nanoparticles was narrow in most cases, with a mean particle diameter under $160 \mathrm{~nm}$. According to reported studies, ${ }^{27,28}$ nanoparticles larger than $200 \mathrm{~nm}$ can be filtered in the spleen. Thus, the nanoparticle dimensions achieved were in the desirable range $100-200 \mathrm{~nm}$ to allow long circulation halflives.

The statistical analysis showed that the PLGA concentration has a significant effect in controlling the particle size and drug entrapment $(p<0.1$, Table $\mathrm{S} 1 \dagger)$. In formulations with a low content of PLGA F1, F3, F5 and F7, the particle size was found to be smaller than in formulations prepared with a high content of PLGA, confirming the importance of PLGA polymer concentration in the emulsification process. This result is in agreement with some previous results ${ }^{5}$ and is attributed to an increase of the viscosity with PLGA concentration. This reduces the net shear stress and results in the formation of droplets with larger size. In addition, the increased viscosity would retard the migration of PLGA solution toward the aqueous phase, resulting in larger droplets that would render larger nanoparticles after EA evaporation. ${ }^{29}$

The zeta potentials (ZP) of all PLGA formulations were negative with values ranging from $-16.1 \mathrm{mV}$ to $-33.2 \mathrm{mV}$ approximately. The negative surface charge is attributed to the presence of free carboxylic acid groups at the chain ends of the PLGA RG504 polymer exposed in the nanoparticle surfaces. According to statistical analysis (Table S1 $\dagger$ ), the PLGA content has not a significant effect in the $\mathrm{ZP}$ value. In addition, the relatively high surface charge indicates that the NPs would be well dispersed in the aqueous medium with good stability and negligible aggregation.

Table 2 Experimental factor and response values in the factorial experimental design

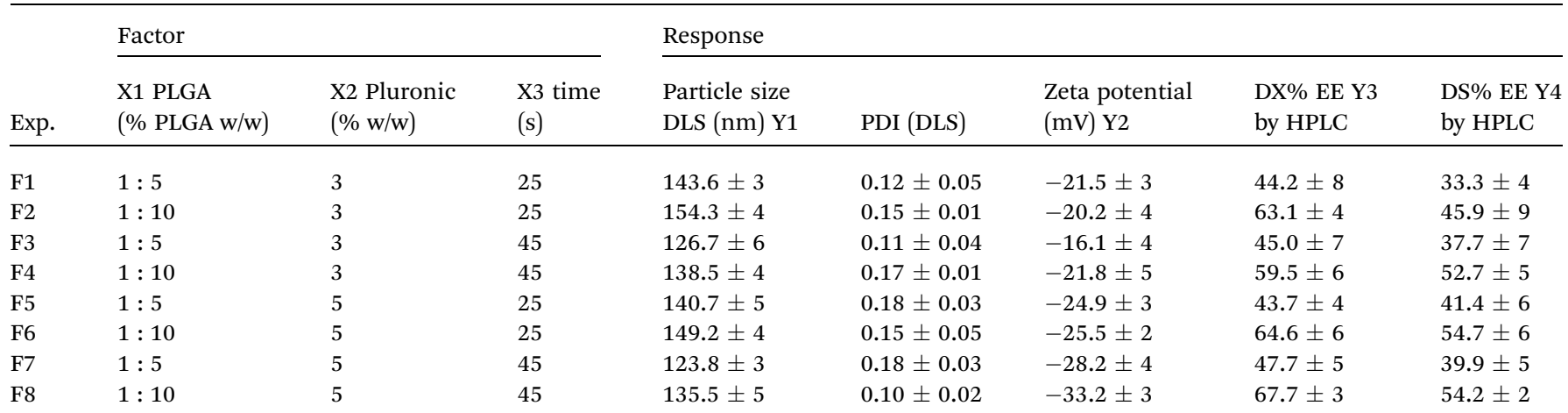


The aforementioned results suggest that the modified single emulsion method assisted by the use of co-solvents can be a suitable, simpler alternative to the double emulsion method, commonly used when co-encapsulating drugs of different polarities. In fact, when ethyl acetate or methanol were used as single solvents in the single emulsification method, no dual encapsulation was achieved (not shown in this work). As shown in Table 2, drug entrapment efficiencies up to around $60 \%$ were obtained using co-solvents for some of the conditions used. The ranges of encapsulation efficiencies (EE) of the different formulations produced by $\mathrm{O} / \mathrm{W}$ emulsification varied from 44 to $67 \%$ and from 37 to $54 \%$ for DX and DS, respectively. The EE of DX in formulations was higher than that obtained for DS due to inherent hydrophobic nature of the former. In general, higher amounts of PLGA (F2, F4, F6 and F8) induced higher EEs, as could be expected. The best overall encapsulation efficiency for both drugs, DX and DS, was achieved in sample F8, presenting an entrapment efficiency of $67.7 \pm 3 \%$ for DX and $54.2 \pm 2 \%$ for DS $(n=3)$, see Table 2 . The tendency observed between PLGA content and drug entrapment can be rationalized by the increased viscosity of the organic phase that leads to an increased diffusional resistance. This retards the diffusion of the drug molecules from the organic phase to the aqueous one during the solvent evaporation step, thereby entrapping more drug in the polymeric nanoparticles as has been previously demonstrated. ${ }^{30}$ The drug loading (DL) achieved in F8 was comparable to the DL accomplished by the formulations where DS and DX were individually encapsulated. Thus, the drug loading of DS and DX in F8 was $2.4 \pm 0.4 \%$ and $4.7 \pm 0.3 \%$, respectively; whereas the individual drug loadings were $2.1 \pm$ $0.8 \%$ (DX) and $4.2 \pm 0.5 \%$ (DS). This shows that the coencapsulation of both drugs was efficiently produced.

The drug encapsulation efficiencies obtained in this work compare favorably with previously published results under similar conditions for single drug entrapment. Thus, Tunçay et $a l .{ }^{31}$ prepared PLGA $(50: 50)$ microspheres incorporating DS by $\mathrm{O} / \mathrm{W}$ emulsification-solvent evaporation method. Polyvinyl alcohol (PVA) and sodium oleate (SO) were used as stabilizers. The authors found a $12.7 \%$ drug content for the formulation prepared with PLGA of $34 \mathrm{kDa}$, and $16.1 \%$ with PLGA of $88 \mathrm{kDa}$. In other studies, Cooper et al. $^{32}$ synthesized PLGA NPs containing DS using an emulsion-diffusion-evaporation technique. The drug entrapment reached $77.3 \pm 3.5 \%$ and $80.2 \pm$ $1.2 \%$ efficiency with the stabilizers didodecyldimethylammonium bromide (DMAB) and PVA, respectively. PLGA NPs containing DX embedded in alginate hydrogel (HG) matrices were elaborated by Kim et al., ${ }^{18}$ using a solvent evaporation technique with mean particle sizes ranging from 400 to $600 \mathrm{~nm}$. The amount of DX loaded in those NPs was estimated as an EE of 79 $\pm 5 \mathrm{wt} \%$. Considering other drug-polymer loading techniques different from emulsification, Campos et al. ${ }^{33}$ prepared PLGA nanoparticles containing dexamethasone acetate by a nanoprecipitation technique; the drug encapsulation efficiency was, in this case, $48 \mathrm{wt} \%$.

Finally, the EEs obtained in this work are above other reported EE values achieved by combined encapsulation of therapeutic drugs. Thus, Niwa et al. ${ }^{34}$ prepared PLGA nanospheres
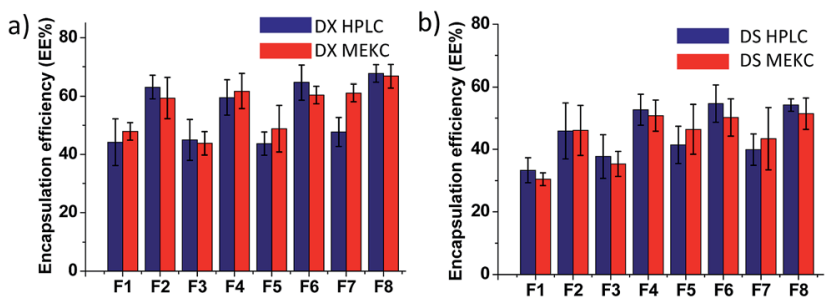

Fig. 1 Encapsulation efficiency by HPLC and MEKC for (a) diclofenac sodium and (b) dexamethasone.

in which they simultaneously loaded water soluble (5-fluorouracil) and insoluble drugs (indomethacin). The authors employed a modified emulsion-solvent diffusion technique. The EEs were, in this case, $5.85 \%$ and $2.65 \%$ for indomethacin and 5-fluorouracil, respectively. NPs of chitosan (CS) and cyclodextrin (CD) loaded simultaneously were prepared via a cross-linking method and methotrexate (MTX) and calcium folinate $(\mathrm{CaF})$ were selected as model drugs of different polarities. The resulting CS/CD nanoparticles showed an EE of $2.48 \pm$ $0.07 \%$ for MTX and $2.64 \pm 0.18 \%$ for CaF. ${ }^{35}$ Song et al. reported entrapment efficiencies of $92.84 \pm 3.4 \%$ for vincristine sulfate and $32.66 \pm 2.9 \%$ for quercetin, hydrophilic and hydrophobic molecules, respectively. ${ }^{5}$

\subsection{Encapsulation efficiency validated by micellar electrokinetic chromatography technique}

To accurately determine the concentration of several drugs in the presence of solvents and other organic molecules is often challenging. As already mentioned, the encapsulation of DX and DS was determined in this work by two independent quantitative methods: MEKC and HPLC (Fig. 1). The amount of DX/DC entrapped in the nanoparticles was also corroborated by the MEKC method after nanoparticle dissolution in $0.1 \mathrm{M}$ $\mathrm{NaOH}$. The encapsulation efficiency is shown in Table S3. $\uparrow$ The ranges of encapsulation efficiency of the different formulations varied from 31 to $51 \%$ and from 33 to $69 \%$ for DS and DX, respectively. The \% EE of dexamethasone in all formulations was higher than that obtained for diclofenac sodium due to inherent hydrophobic nature of the former. The results reported in Table S3† show that, while both quantification techniques display the same \% EE trends, the specific results may vary in some cases. This can be attributed to the possibility that, even after washing, part of the drug could still be adsorbed on the surface of the nanoparticles, leading to slight differences for both techniques.

\subsection{Characterization of the optimal DS-DX nanoparticle formulation}

Particle morphology by transmission electronic microscopy. The morphology of the optimized DX-DS/PLGA NP8 formulation and non-loaded blank (B-NP) were observed by transmission electron microscopy (TEM) (Fig. 2a and b). The TEM micrographs show that both nanoparticles present spherical morphology without evidence of particle aggregation. The mean 

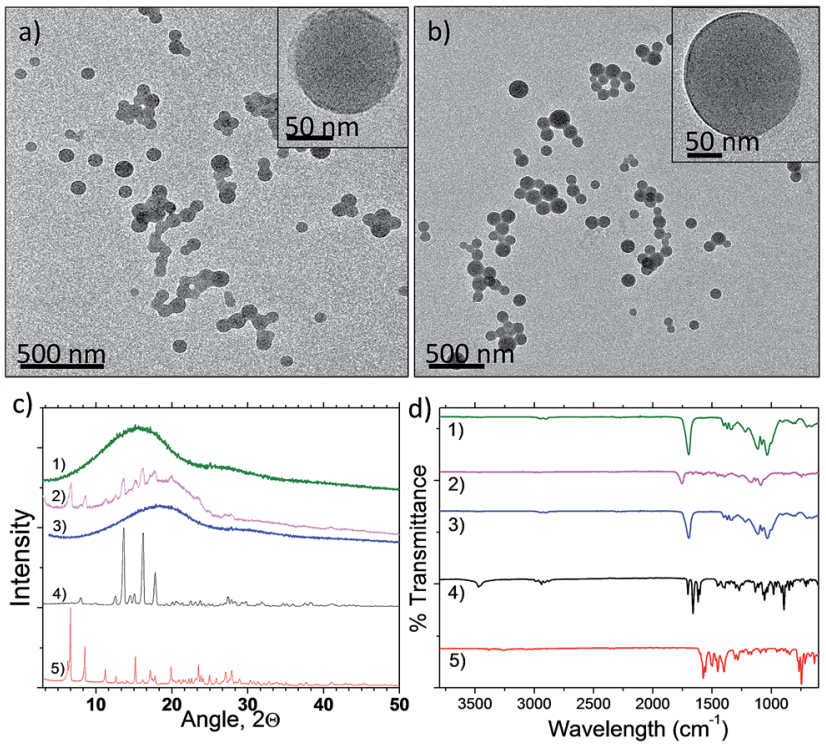

Fig. 2 TEM image of (a) diclofenac sodium-dexamethasone loaded PLGA nanoparticles (with magnification) and (b) non-drug loaded NPs (with magnification). (c) and (d) XRD diffractograms and FT-IR spectra: (1) F8, (2) physical mixture (DX, DS and PLGA), (3) PLGA and (4) dexametasone and (5) diclofenac sodium.

particle size of the DS-DX loaded and blank NPs formulations were $129.1 \pm 10.3 \mathrm{~nm}$ and $135.9 \pm 15.2 \mathrm{~nm}$, respectively. The sizes obtained from the TEM measurements are in good agreement with the results obtained from the SEM and DLS measurements. According to the presented results, the dual encapsulation of DS and DX did not alter significantly the PLGA nanoparticle dimensions.

X-ray diffractometry. XRD diffractograms of pure PLGA polymer and drugs (DX and DS) were compared with the physical mixture and with loaded DX-DS PLGA NPs (Fig. 2c). The Xray diffraction pattern of pure drugs, DX and DS, exhibited crystalline nature by numerous sharp diffraction peaks. However, these characteristic peaks were not observed in DXDS/PLGA-NPs (F8). This difference hypothesized that DX and DS were present mainly as an amorphous or disordered-crystalline state inside the PLGA nanoparticles. ${ }^{36}$ Also it is well known that small nanocrystals do not produce measurable diffraction peaks due to the limitations of conventional diffractometers. DS and DX could be encapsulated in the dissolved state, in form of molecular dispersion, because the encapsulation of both molecules was confirmed by HPLC and MEKC. This hypothesis was confirmed by the XRD analysis of the physical mixture PLGA and similar concentration of DS and DX, since in this analysis the typical DS and DX crystalline peaks were observed. No peaks were observed for PLGA either in the formulation or as raw polymer, in agreement with the fact that PLGA is an amorphous copolymer.

Fourier-transform infrared spectroscopy (FT-IR). FT-IR spectra of DS, DX, PLGA, DS-DX-loaded PLGA-NPs and the physical mixture (DS-DX-PLGA) are shown in Fig. 2d. The DX spectrum shows the characteristic absorption bands at 3390 and $1268 \mathrm{~cm}^{-1}$ attributed to the stretching vibration of $\mathrm{O}-\mathrm{H}$ and
C-F bonds, respectively; the stretching vibration at 1706, 1662 and $1621 \mathrm{~cm}^{-1}$ were attributed to $-\mathrm{C}=\mathrm{O}$ and double bond framework conjugated to $-\mathrm{C}=\mathrm{O}-$, in agreement with other studies. ${ }^{37}$ The DS spectrum depicts a characteristic peak at 3380 $\mathrm{cm}^{-1}$ due to the $\mathrm{N}-\mathrm{H}$ stretching frequency of the secondary amine. The transmittance bands at 1310 and $1284 \mathrm{~cm}^{-1}$ are attributed to the $\mathrm{C}-\mathrm{N}$ stretching and the peaks at 1550 and 1570 $\mathrm{cm}^{-1}$ were attributed to theo $\mathrm{C}=\mathrm{C}$ stretching and $\mathrm{C}=\mathrm{O}$ stretching of the carboxylate groups, respectively. ${ }^{37}$

The spectrum obtained for the physical mixture showed characteristic transmittance bands observed for each of the components of the mixture separately (DX, DS and PLGA) with a shift and broadening which can be attributed to drug-polymer interactions in the solid dispersions. This shift suggests that polymer-drug interactions prevail over drug-drug interactions. DX-DS PLGA NPs did not show any chemical bonds besides the characteristic of the parent PLGA. This was attributed to the correct encapsulation within the polymeric matrix and that the stronger signals of the chemical bonds originated from the PLGA carrier could mask the IR signals produced by the reduced amount of the encapsulated drugs when hosted as molecular dispersions.

Drug delivery and kinetics. Drug release kinetics of F8 in phosphate buffer solution was studied during $72 \mathrm{~h}$. Each sample was analyzed in triplicate (Fig. 3a). Fig. 3a shows an initial burst release during the first $5 \mathrm{~h}$ of incubation for both drugs. This burst release has been attributed to either nonencapsulated drug molecules on the surface of PLGA NPs or drug molecules close to the surface (immersed in the polymer matrix) but easy accessible after the hydration of the polymer. ${ }^{38}$ Then, a slow and continuous release was observed for the next $48 \mathrm{~h}$. The release of DX was slower, with $60 \%$ of the drug release up to $72 \mathrm{~h}$ in comparison with a $80 \%$ released obtained for DS in the same time.

The cumulative drug release data were fitted into different release models (i.e., zero order, first order, Higuchi's square root plot and Hixson-Crowell cube root plot ${ }^{39}$ ). The best model was selected according to the correlation coefficient $(r)$ determined from the linear regression fit for each model.

Applying the aforementioned models, the correlation coefficients show that the release of DX and DS from DX-DS/NPs followed the Higuchi model $\left(r_{\mathrm{DX}}{ }^{2}=0.975\right.$ and $\left.r_{\mathrm{DS}}{ }^{2}=0.968\right)$ (Fig. 3b). This indicates that the drug release from the polymeric

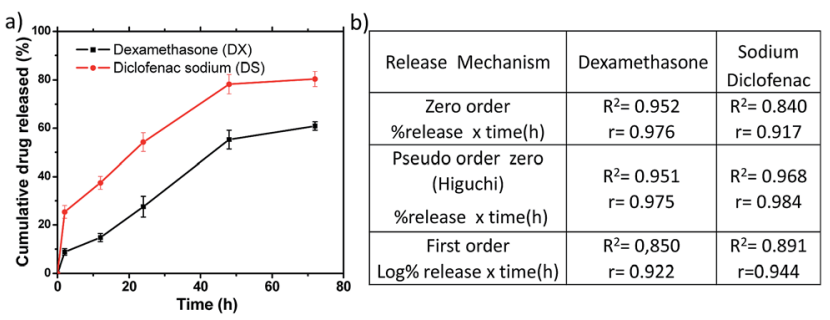

Fig. 3 (a) In vitro release profile of DX and DS in $\mathrm{pH} 7.4$ phosphate buffer (mean $\pm S D, n=3$ ) from DX-DS/NPs. (b) Kinetic study of release of DX and DS from F8. Correlation coefficients $(r)$ of mathematical models fitting the release kinetics. 


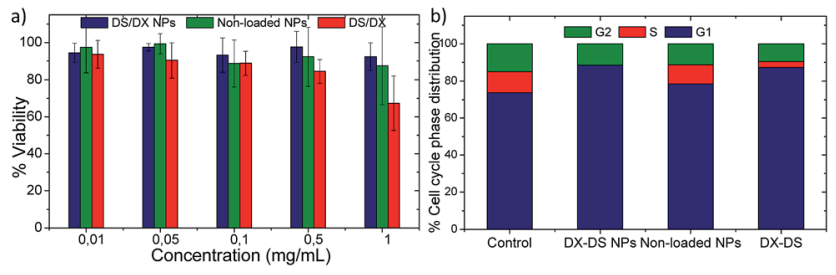

Fig. 4 (a) Cell viability of free DX and DS, DX-DS/NPs and non-loaded NPs on human dermal fibroblasts. Percentages are displayed as mean $\pm \mathrm{SD}(n=5)$. (b) Cell cycle distribution in human dermal fibroblasts in the experimental groups assayed: control group (not treated cells), DX-DS/NPs, non-loaded NPs and free DS and DX.

nanoparticles was governed by diffusion and a dissolutioncontrolled process from the polymeric matrix rather than a PLGA degradation-driven controlled process in agreement with some recent results. ${ }^{40}$ In addition, the reported degradation of PLGA heteropolymer is approximately 2-6 weeks, which confirms that the kinetic model proposed for the DS-DX release is not governed by degradationgoverned by degradation. ${ }^{41}$

\subsection{In vitro biocompatibility studies}

To determine the in vitro biocompatibility of the F8 formulation, its effects regarding cellular metabolism, membrane and nucleus in human dermal fibroblasts were studied. The effects in the cell metabolism were analyzed by the Alamar Blue assay (Fig. 4a) at different concentrations (0.01-1 $\mathrm{mg} \mathrm{mL} \mathrm{m}^{-1}$ ). In general, the increase in NPs or drugs concentration did not show statistically significant differences in cell viability. According to the ISO 10993-5, in which a reduction in viability higher than $30 \%$ is considered a cytotoxic effect, our data showed that the subcytotoxic concentration was $1 \mathrm{mg} \mathrm{mL}^{-1}$ which was the concentration considered in further studies.

Previous studies have reported no cytotoxic effects of different formulations of PLGA NPs, such as $\alpha$-elastin- $g$-PLGA NPs though DX loaded elastin-g-PLGA NPs exhibited a reduction in viability of $50 \%$ and free DX of $70 \%$ at a concentration of 50 $\mu \mathrm{g} \mathrm{mL}^{-1}$ in human umbilical artery smooth muscle cells ${ }^{42}$ which is not in accordance with our results in which, at the same concentration, viability was higher than $90 \%$ in the three experimental groups assayed. The treatment of other cell types with free DX has displayed different results though the reduction in viability has been clearly shown. ${ }^{43-45}$ Other authors have reported the high cytotoxicity of DS loaded in PLGA/PEG scaffolds (1 $\mathrm{mg}$ per scaffold) in mouse primary calvarial osteoblasts, ${ }^{46}$ as well as free DS in human microvascular endothelial cells at concentrations lower than ours $(0.1 \mathrm{mM})^{47}$ while other studies have revealed similar viability percentages at low concentrations (up to $100 \mu \mathrm{M}$ ) though showing differences between different cell lines displaying an enhanced toxic effect in somatic $v s$. tumor cells ${ }^{48}$ or even at higher concentrations $\left(3 \mathrm{mg} \mathrm{mL}{ }^{-1}\right.$ ) in primary rat embryo fibroblasts. ${ }^{49}$

Cell membrane damage after treatment with the considered subcytotoxic concentration $\left(0.5 \mathrm{mg} \mathrm{mL}^{-1}\right)$ of NPs or drugs was evaluated by flow cytometry through the distribution of viability, apoptosis and necrosis (Table 3). Viability was not
Table 3 Apoptosis evaluation after treatment with NPs or drugs. Control group is the reference sample (not treated cells)

\begin{tabular}{lllll}
\hline Phases & Control & DX-DS/NPs & $\begin{array}{l}\text { Non-loaded } \\
\text { NPs }\end{array}$ & DX-DS \\
\hline Necrosis & $0.8 \%$ & $0.9 \%$ & $0.2 \%$ & $0.2 \%$ \\
Late apoptosis & $4.8 \%$ & $10.5 \%$ & $5.5 \%$ & $5.6 \%$ \\
Early apoptosis & $24.6 \%$ & $28.2 \%$ & $13.8 \%$ & $14.8 \%$ \\
Viability & $69.8 \%$ & $60.4 \%$ & $80.5 \%$ & $79.3 \%$ \\
\hline
\end{tabular}

significantly affected; in fact only drug loaded NPs exerted a slight decrease (9.4\%). Accordingly, apoptosis displayed a low increase (9\%) in drug loaded NPs.

Thus, cell treatment with NPs or drugs did not show any harmful effect on cell membrane. In this sense, previous studies have shown the induction of apoptosis in multiple myeloma cells by DX mediated by the glucocorticoid response element transactivation ${ }^{50}$ as well as in other cell types such as activated T-cells ${ }^{51}$ or mice thymocytes at a dose of $1 \mathrm{mg}$ per mouse, ${ }^{52}$ according to the well-known apoptotic effects of glucocorticoids. ${ }^{53}$ Furthermore, rat colonocytes obtained from an in vivo model after treatment with curcumin and DS also developed apoptosis ${ }^{54}$ and it has been found apoptotic effects in hepatocytes $^{55}$ and in different tumor cells ${ }^{56,57}$ after DS treatment.

The effect of NPs and drugs treatment on fibroblasts cell cycle is shown in Fig. $4 \mathrm{~b}$. The presence of DX and DS reduced S phase being more accentuated in the drug loaded NPs group in which $\mathrm{S}$ phase is not displayed, that means that DNA replication was halted. In this sense, other authors have studied the effects of DX in cell cycle though their findings were contradictory showing a reduction in $\mathrm{S}$ phase in human cultured airway smooth muscle after treatment with $100 \mathrm{nM}$ of $\mathrm{DX}^{58}$ or even an arrest in G1 in cultured rat aortic smooth muscle cells at concentrations up to $0.1 \mathrm{M},{ }^{59}$ while other authors pointed to an increase in DNA replication in asthmatic fibroblasts at similar concentrations. ${ }^{60}$ These differences may be attributed to the different experimental designs and the different cell lines assayed. On the other hand, DS administration (6 $\mathrm{mg}$ per animal weekly) to an in vivo model has also been shown as able to arrests the cell cycle and inhibit cell proliferation, suggesting antitumorigenic effects, through the reduction in cyclins and cyclin-dependent kinases expression, ${ }^{54}$ which has been also previously reported in DX. ${ }^{58}$ In this sense, glioblastoma cells also displayed a reduction in $\mathrm{S}$ phase after DS treatment $(0.2$ $\mathrm{mM}),{ }^{61}$ pointing again to the potential antitumorigenic effects of diclofenac.

It may be concluded that the loaded NPs here described reduced the cytotoxicity linked to DX and DS while non-loaded NPs did not exert toxic effects and thus their effects regarding cell apoptosis and cell cycle were not significant.

\section{Conclusions}

This work evidences that the w/o emulsification-evaporation technique can be used with a careful selection of the 
appropriate solvents to produce PLGA nanoparticles with in situ simultaneous encapsulation of hydrophobic and hydrophilic drugs (DX and DS). The co-encapsulation of two drugs, DS and DX, with different polarity was successfully achieved thanks to the use of a mixture of methanol and ethyl acetate as the organic phase to increase the solubility of both hydrophilic and hydrophobic drugs. The optimum solvent methanol/ethyl acetate ratio out of the ones studied was $1: 9(\mathrm{v} / \mathrm{v})$. The load of both drugs has been quantitatively determined using the MEKC and HPLC analytical methods, which gave comparable results and the same trends with the experimental variables considered. The most suitable PLGA formulation for an adequate encapsulation of both drugs, DX and DS, was F8 (drug/polymer ratio, $1: 10$; surfactant concentration $5 \% \mathrm{w} / \mathrm{v}$ and sonication time $45 \mathrm{~s}$ ), presenting a drug loading of DS and DX of $2.4 \pm 0.4 \%$ and $4.7 \pm 0.3 \%$, respectively. Compared to previous reports, this study provides high encapsulation efficiency and reduced sizes. DS and DX release profiles can be explained as the outcome of diffusion and dissolutioncontrolled processes from the polymeric matrix rather than through the degradation of PLGA. Furthermore, the cytobiocompatibility of these NPs at the assayed doses has also been demonstrated.

\section{Acknowledgements}

The authors wish to acknowledge EU CIG-Marie Curie under the REA grant agreement no. 321642 and the ERC Consolidator Grant program (ERC-2013-CoG-614715, NANOHEDONISM). The "Fundação de Amparo à Pesquisa do Estado de São Paulo" (FAPESP) and "Conselho Nacional de Desenvolvimento Científico e Tecnológico" (CNPq) for continuous financial support are also gratefully acknowledged. We also thank Eugenio Vispe for his advices and suggestions during the HPLC determination of both drugs.

\section{Notes and references}

1 J. M. Chan, P. M. Valencia, L. F. Zhang, R. Langer and O. C. Farokhzad, Methods Mol. Biol., 2010, 624, 163-175.

2 R. B. Friedrich, M. C. Fontana, R. C. R. Beck, A. R. Pohlmann and S. S. Guterres, Quim. Nova, 2008, 31, 1131-1136.

3 S. Dhar, F. X. Gu, R. Langer, O. C. Farokhzad and S. J. Lippard, Proc. Natl. Acad. Sci. U. S. A., 2008, 105, 17356-17361.

4 Y. Cao, B. C. Wang, Y. Z. Wang and D. S. Lou, J. Pharm. Sci., 2014, 103, 3205-3216.

5 X. R. Song, Y. Zhao, S. X. Hou, F. Y. Xu, R. Zhao, J. Y. He, Z. Cai, Y. B. Li and Q. H. Chen, Eur. J. Pharm. Biopharm., 2008, 69, 445-453.

6 L. F. Zhang, A. F. Radovic-Moreno, F. Alexis, F. X. Gu, P. A. Basto, V. Bagalkot, S. Y. Jon, R. S. Langer and O. C. Farokhzad, ChemMedChem, 2007, 2, 1268-1271.

7 M. Cetin, A. Atila and Y. Kadioglu, AAPS PharmSciTech, 2010, 11, 1250-1256.

8 T. D. Warner, I. Vojnovic, D. Bishop-Bailey and J. A. Mitchell, Faseb J., 2006, 20, 542-544.
9 C. K. Ong, P. Lirk, C. H. Tan and R. A. Seymour, Clin. Med. Res., 2007, 5, 19-34.

10 D. F. Liu, Y. F. Ge, Y. Tang, Y. B. Yuan, Q. Zhang, R. Li and Q. W. Xu, J. Microencapsulation, 2010, 27, 726-734.

11 C. Stahn, M. Lowenberg, D. W. Hommes and F. Buttgereit, Mol. Cell. Endocrinol., 2007, 275, 71-78.

12 X. L. Lu, M. D. Howard, M. Mazik, J. Eldridge, J. J. Rinehart, M. Jay and M. Leggas, AAPS J., 2008, 10, 133-140.

13 D. L. Boyle, J. Moore, L. Yang, L. Sorkin and G. S. Firestein, Arthritis Rheum., 2001, 44, S388.

14 J. Joris, A. Costello, R. Dubner and K. M. Hargreaves, Pain, 1990, 43, 95-103.

15 G. Draisci and M. J. Iadarola, Mol. Brain Res., 1989, 6, 31-37. 16 B. O. Bamgbose, J. A. Akinwande, W. L. Adeyemo, A. L. Ladeinde, G. T. Arotiba and M. O. Ogunlewe, Curr. Ther. Res., 2006, 67, 229-240.

17 J. Buritova, P. Honore, V. Chapman and J. M. Besson, Pain, 1996, 64, 559-568.

18 D. H. Kim and D. C. Martin, Biomaterials, 2006, 27, 30313037.

19 S. Acharya and S. K. Sahoo, Adv. Drug Delivery Rev., 2011, 63, 170-183.

20 X. Y. Xiong, K. C. Tam and L. H. Gan, J. Controlled Release, 2005, 103, 73-82.

21 R. A. Jain, Biomaterials, 2000, 21, 2475-2490.

22 J. Panyam and V. Labhasetwar, Adv. Drug Delivery Rev., 2003, 55, 329-347.

23 R. M. Mainardes and R. C. Evangelista, Int. J. Pharm., 2005, 290, 137-144.

24 N. M. Khalil, T. C. F. do Nascimento, D. M. Casa, L. F. Dalmolin, A. C. de Mattos, I. Hoss, M. A. Romano and R. M. Mainardes, Colloids Surf., B, 2013, 101, 353-360.

25 C. Fillafer, D. S. Friedl, M. Wirth and F. Gabor, Small, 2008, 4, 627-633.

26 M. T. H. Mosavian and A. Hassani, J. Dispersion Sci. Technol., 2010, 31, 293-298.

27 S. Stolnik, L. Illum and S. S. Davis, Adv. Drug Delivery Rev., 2012, 64, 290-301.

28 Y. Tabata and Y. Ikada, Adv. Polym. Sci., 1990, 94, 107-141.

29 H. Y. Kwon, J. Y. Lee, S. W. Choi, Y. S. Jang and J. H. Kim, Colloids Surf., A, 2001, 182, 123-130.

30 A. Budhian, S. J. Siegel and K. I. Winey, Int. J. Pharm., 2007, 336, 367-375.

31 M. Tuncay, S. Calis, H. S. Kas, M. T. Ercan, I. Peksoy and A. A. Hincal, Int. J. Pharm., 2000, 195, 179-188.

32 D. L. Cooper and S. Harirforoosh, PLoS One, 2014, 9, e87326. 33 I. M. F. Campos, T. M. Santos, G. M. F. Cunha, K. M. M. N. Silva, R. Z. Domingues, A. D. Cunha and K. C. D. Figueiredo, J. Appl. Polym. Sci., 2014, 131, 41199.

34 T. Niwa, H. Takeuchi, T. Hino, N. Kunou and Y. Kawashima, J. Controlled Release, 1993, 25, 89-98.

35 J. G. Ji, S. L. Hao, W. Q. Liu, D. J. Wu, T. F. Wang and Y. Xu, Colloids Surf., B, 2011, 83, 103-107.

36 M. Guada, V. Sebastian, S. Irusta, E. Feijoo, M. D. DiosVieitez and M. J. Blanco-Prieto, Int. J. Nanomed., 2015, 10, 6541-6553. 
37 L. B. Rodrigues, H. F. Leite, M. I. Yoshida, J. B. Saliba, A. S. Cunha and A. A. G. Faraco, Int. J. Pharm., 2009, 368, 1-6.

38 J. Wang, B. A. Wang and S. P. Schwendeman, J. Controlled Release, 2002, 82, 289-307.

39 M. A. Akl, A. Kartal-Hodzic, T. Oksanen, H. R. Ismael, M. M. Afouna, M. Yliperttula, A. M. Samy and T. Viitala, J. Drug Delivery Sci. Technol., 2016, 32, 10-20.

40 N. Silveira, M. M. Longuinho, S. G. Leitao, R. S. F. Silva, M. C. Lourenco, P. E. A. Silva, M. D. F. R. Pinto, L. G. Abracado and P. V. Finotelli, Mater. Sci. Eng., C, 2016, 58, 458-466.

$41 \mathrm{H}$. M. Mansour, M. Sohn, A. Al-Ghananeem and P. P. DeLuca, Int. J. Mol. Sci., 2010, 11, 3298-3322.

42 F. S. Palumbo, S. Rigogliuso, G. Ghersi, G. Pitarresi, C. Fiorica, M. Di Stefano and G. Giammona, Mol. Pharmaceutics, 2014, 11, 651.

43 W. Suwanjang, A. Y. Abramov, P. Govitrapong and B. Chetsawang, J. Steroid Biochem., 2013, 138, 116-122.

44 M. W. N. Wong, W. T. Lui, S. C. Fu and K. M. Lee, Acta Orthop., 2009, 80, 363-367.

45 Y. Zhao, Y. Zuo, H. J. Huo, Y. L. Xiao, X. J. Yang and D. Q. Xin, Mol. Med. Rep., 2014, 9, 923-927.

46 L. E. Sidney, T. R. J. Heathman, E. R. Britchford, A. Abed, C. V. Rahman and L. D. K. Buttery, Tissue Eng., Part A, 2015, 21, 362-373.

47 A. Wiktorowska-Owczarek, Acta Pharm., 2014, 64, 131-138. 48 J. Schmidt, F. M. Klingler, E. Proschak, D. Steinhilber, M. Schubert-Zsilavecz and D. Merk, Sci. Rep., 2015, 5, 14782.

49 M. S. M. Al-Nimer, H. G. Hameed and M. M. Mahmood, Saudi Pharm. J., 2015, 23, 483-486.
50 S. Sharma and A. Lichtenstein, Blood, 2008, 112, 1338-1345. 51 M. S. G. Montani, L. Tuosto, R. Giliberti, L. Stefanini, E. Cundari and E. Piccolella, Cell Death Differ., 1999, 6, 7986.

52 V. Chmielewski, F. Drupt and R. Morfin, Immunol. Cell Biol., 2000, 78, 238-246.

53 S. Greenstein, K. Ghias, N. L. Krett and S. T. Rosen, Clin. Cancer Res., 2002, 8, 1681-1694.

54 C. Rana, H. Piplani, V. Vaish, B. Nehru and S. N. Sanyal, Tumor Biol., 2015, 36, 5999-6010.

55 M. J. Gomez-Lechon, X. Ponsoda, E. O'Connor, T. Donato, R. Jover and J. V. Castell, Toxicol. In Vitro, 2003, 17, 675-680.

56 F. Cecere, A. Iuliano, F. Albano, C. Zappelli, I. Castellano, P. Grimaldi, M. Masullo, E. De Vendittis and M. R. Ruocco, J. Biomed. Biotechnol., 2010, 801726.

57 L. Fredriksson, B. Herpers, G. Benedetti, Q. Matadin, J. C. Puigvert, H. de Bont, S. Dragovic, N. P. E. Vermeulen, J. N. M. Commandeur, E. Danen, M. de Graauw and B. van de Water, Hepatology, 2011, 53, 2027-2041.

58 D. Fernandes, E. Guida, V. Koutsoubos, T. Harris, P. Vadiveloo, J. W. Wilson and A. G. Stewart, Am. J. Respir. Cell Mol. Biol., 1999, 21, 77-88.

59 T. D. Reil, R. Sarkar, V. S. Kashyap, M. Sarkar and H. A. Gelabert, J. Surg. Res., 1999, 85, 109-114.

60 B. Fouty, T. Moss, V. Solodushko and M. Kraft, Eur. Respir. J., 2006, 27, 1160-1167.

61 V. Leidgens, C. Seliger, B. Jachnik, T. Welz, P. Leukel, A. Vollmann-Zwerenz, U. Bogdahn, M. Kreutz, O. M. Grauer and P. Hau, PLoS One, 2015, 10, e0140613. 